\title{
Genetic Ablation of Neural Progenitor Cells Impairs Acquisition of Trace Eyeblink Conditioning
}

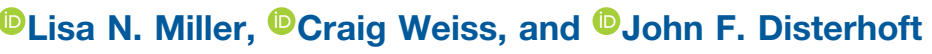 \\ https://doi.org/10.1523/ENEURO.0251-19.2019 \\ Department of Physiology, Feinberg School of Medicine, Northwestern University, Chicago, Illinois 60611
}

\begin{abstract}
Adult-born neurons are believed to play a role in memory formation by providing enhanced plasticity to the hippocampus. Past studies have demonstrated that reduction of neurogenesis impairs associative learning, but these experiments used irradiation or neurotoxic substances, which may have had unintended off-target effects. Therefore, to investigate the role of these adult-born neurons more precisely, we used nestin-HSV-TK transgenic mice (Nes-TK) to selectively ablate newborn neurons. Nes-TK mice were fed a chow infused with valganciclovir to induce the ablation of neural progenitor cells. After being on this diet for 4 weeks, mice were trained on trace eyeblink conditioning, a hippocampus-dependent temporal associative memory task. Following the completion of training, brain sections from these animals were stained for doublecortin, a marker for immature neurons, to quantify levels of neurogenesis. We found that male transgenic mice on valganciclovir had significantly decreased amounts of doublecortin relative to male control animals, indicating a successful reduction in levels of neurogenesis. In conjunction with this reduction in neurogenesis, the male transgenic mice on valganciclovir learned at a significantly slower rate than male control mice. The female Nes-TK mice on valganciclovir showed no significant decrease in neurogenesis and no behavioral impairment relative to female control mice. Ultimately, the results are consistent with, and expand upon, prior studies that demonstrated that adult-born neurons are involved in the formation of associative memories. This study also provides a foundation to continue to explore the physiological role of newborn neurons with in vivo recordings during behavioral training.
\end{abstract}

Key words: dentate gyrus; learning; memory; neurogenesis; trace eyeblink conditioning

\section{Significance Statement}

Newborn neurons in the adult brain have been shown to be involved in associative learning, but many prior studies illustrating this point used neurotoxins or irradiation to ablate newborn neurons, which may have had unintended off-target effects. Therefore, we used a transgenic mouse model to eliminate adult-born neurons in a more controlled, precise manner. Ultimately, we demonstrate that the reduction of neurogenesis leads to an impairment in learning in males, and that levels of neurogenesis are associated with the rate of learning and overall performance on trace eyeblink conditioning.

\section{Introduction}

Neurogenesis in the adult brain occurs in the dentate gyrus (DG) and produces new neurons that mature into granule cells and integrate into existing circuitry (Altman

Received June 28, 2019; accepted August 23, 2019; First published September 16, 2019

The authors declare no competing financial interests.

Author contributions: L.N.M., C.W., and J.F.D. designed research; L.N.M. performed research; L.N.M. analyzed data; L.N.M., C.W., and J.F.D. wrote the paper. and Das, 1965; Dayer et al., 2003). These highly excitable neural progenitor cells are believed to play a role in memory formation by providing enhanced plasticity to the hippocampus (Snyder et al., 2001; Mongiat et al., 2009; 
Suter et al., 2019). Indeed, there are some studies that have found that reducing the number of newborn neurons impairs memory acquisition on different associative memory tasks. Specifically, the ablation of newborn neurons through systemic administration of an antimitotic agent prevented male rats from learning trace eyeblink conditioning (tEBC; Shors et al., 2001). Additionally, the elimination of adult-born neurons in male rats through fractionated irradiation led to an impairment in the hippocampal-dependent place recognition test, but had no effect on the hippocampal-independent object recognition task (M'Harzi et al., 1991; Steckler et al., 1998; Madsen et al., 2003). However, there have also been conflicting results depending on the species and methodology used to reduce neurogenesis. Snyder et al. (2005) observed that eliminating newborn neurons through irradiation did not impact acquisition on the hippocampaldependent Morris water maze (MWM), but did impair long-term memory on this task. However, a study in male mice failed to see an impact of ablating newborn neurons on MWM performance (Saxe et al., 2006). Furthermore, while systemic administration of an antimitotic agent reduced freezing in male rats during trace, but not contextual, fear conditioning (Shors et al., 2002), the elimination of newborn neurons through irradiation and genetic manipulations in male mice led to a reduction in freezing behavior during contextual, but not trace, fear conditioning (Saxe et al., 2006). These inconsistencies therefore warrant further investigation into the involvement of adultborn neurons in associative learning.

A limitation of these past studies is that the large majority of them made use of irradiation or neurotoxic substances that may have had unintended off-target effects. For example, the antimitotic agent methylazoxymethanol acetate (MAM) used in some studies has been shown to impact the overall health of an animal and to induce hypoactivity (Dupret et al., 2005). With newer genetic techniques, however, we can investigate whether adultborn neurons are necessary for the acquisition of associative learning with greater precision and fewer potential confounds. Specifically, we used nestin-HSV-TK transgenic mice (Nes-TK) to selectively reduce the number of newborn neurons to investigate the role of these neural progenitor cells (Yu et al., 2008). These mice express a modified herpes simplex virus thymidine kinase driven by a nestin promoter and its second intron regulatory element, which allows for temporally regulated induced ablation of dividing neural progenitors through systemic administration of ganciclovir or its prodrug valganciclovir (Val; Yu et al., 2008; Mustroph et al., 2015).

Acknowledgments: We would like to thank Amy Rapp, Mike McCarthy, and Dr. Matthew Oh for technical support and general feedback.

Correspondence should be addressed to Lisa N. Miller at lisamiller2021@ u.northwestern.edu or John F. Disterhoft at Jdisterhoft@northwestern.edu.

https://doi.org/10.1523/ENEURO.0251-19.2019

Copyright (C) 2019 Miller et al.

This is an open-access article distributed under the terms of the Creative Commons Attribution 4.0 International license, which permits unrestricted use, distribution and reproduction in any medium provided that the original work is properly attributed.
To study whether adult-born neurons are necessary for learning, we trained animals on tEBC, a hippocampaldependent temporal associative memory task in which an otherwise neutral conditioned stimulus (CS) is paired with an aversive unconditioned stimulus (US) that causes a reflexive eyeblink response. The two stimuli are separated in time by a stimulus-free trace interval, and animals learn to associate the two stimuli over many trials. On learning this association, animals start to close their eye during the trace interval in anticipation of the US, which is known as the conditioned response (CR). The advantages of tEBC are that it takes many trials for animals to learn, which allows for comparisons of the rate of learning, as well as the ability to look at changes in cellular activity over the course of learning through in vivo recording methods.

The goal of this study was to address whether genetic ablation of neural progenitor cells affects the acquisition of tEBC, using both male and female mice. Ultimately, we found that a reduction in the number of newborn neurons impairs the acquisition of this hippocampus-dependent temporal learning task, and that levels of neurogenesis are correlated with overall performance and rate of learning in male mice.

\section{Materials and Methods}

\section{Animals}

Animal care procedures were conducted in accordance with National Institutes of Health guidelines and as approved by the Northwestern University Institutional Animal Care and Use Committee. The Nes-TK transgenic mouse line (stock \#029671, The Jackson Laboratory; RRID:IMSR_JAX:029671) was originally developed in the laboratory of S.G. Kernie (Columbia University, New York, NY; Yu et al., 2008). Mice were bred in a Northwestern University animal facility, and the genotype of each animal was determined by a tail snip sample sent to Transnetyx. Both male and female mice were used in this study, and the estrous cycle was not monitored (Prendergast et al., 2014; Fritz et al., 2017).

Four weeks before behavioral training, at $\sim 8-14$ weeks of age, mice were singly housed and provided ad libitum access to either regular chow or chow infused with valganciclovir (1350 mg/kg; Custom Animal Diets), a valine ester prodrug of ganciclovir. This schedule was based on the findings from the study by Shors et al. (2001) that demonstrated that newborn neurons in rats are $\sim 1-2$ weeks old when they become involved in learning tEBC. However, there is a 1-2 week delay in the maturation of young granule cells in mice compared with rats (Snyder et al., 2009), which is why mice were started on their given diet 4 weeks before tEBC. Assigned diets were maintained until the animals were killed. The experimental group consisted of Nes-TK mice eating Val-chow, and the three control groups included Nes-TK mice eating regular chow, wild-type (WT) mice eating Val-chow, and WT mice eating regular chow. These control groups were used to investigate whether the drug or genotype alone would have an effect on learning. Ultimately, after there was no observed difference in learning among the three groups, they were combined into one control group to avoid using 
animals unnecessarily. Val-chow was weighed weekly to monitor food intake to calculate average Val dosage.

\section{Trace eyeblink conditioning}

Two weeks before the start of behavioral training, mice underwent headbolt implantation surgery, during which subdermal wires were placed around the orbicularis oculi muscle to measure eyeblink response via electromyography (EMG) activity. After 1 week of recovery, mice were handled for $5 \mathrm{~min} / \mathrm{d}$ for $3 \mathrm{~d}$ and then habituated to the training chamber for $2 \mathrm{~d}$, for $\sim 45-60 \mathrm{~min} / \mathrm{d}$. Finally, animals were trained two at a time on tEBC for $10 \mathrm{~d}$.

During $\mathrm{tEBC}$, mice were head-fixed atop a freely rotating cylinder (Heiney et al., 2014; Lin et al., 2016). Each session was composed of 40 trials, each consisting of a $250 \mathrm{~ms}$ whisker displacement (i.e., CS; $\pm 50 \mu \mathrm{m}$ at $62 \mathrm{~Hz}$, delivered by a comb attached to a piezo actuator) paired with a $30 \mathrm{~ms}$ air puff to the cornea (i.e., US; $3.5 \mathrm{psi}$, delivered by a blunted 16 gauge needle pointed at the eye). Presenting the CS and US to different sensory modalities prompts learning that requires the integration of sensory information at higher-level cortical structures. This is in contrast to other trace-conditioning paradigms where the CS and US are both applied to the whisker pad (Troncoso et al., 2004), which may be mediated by motor neurons or the brainstem. The two stimuli were separated by a $250 \mathrm{~ms}$ stimulus-free trace interval, and the mean intertrial interval was $45 \mathrm{~s}$ (range, $30-60 \mathrm{~s}$ ). White noise $(78-80 \mathrm{~dB})$ was played for the duration of each session. Air pressure and vibration intensity were calibrated between each set of animals using a manometer (Thermo Fisher Scientific) and a displacement sensor (optoNCDT 1320, Micro-Epsilon), respectively. The displacement sensor also provided real-time confirmation that the whisker vibration was delivered during each trial. Custom LabView software was used to control stimuli presentation, data collection, and data analysis. CRs were defined as EMG activity during the $200 \mathrm{~ms}$ before US presentation that was $>4$ SDs above baseline for $>15 \mathrm{~ms}$, with the baseline being defined as the 250 ms before CS onset. An animal was considered to reach the learning criterion when it demonstrated CRs for at least $60 \%$ of trials within a session.

\section{Immunohistochemistry}

Two weeks after the completion of training, mice underwent an intracardial perfusion with $0.1 \mathrm{M}$ PBS followed by $4 \%$ paraformaldehyde (PFA). The brain was removed and stored in $4 \%$ PFA overnight at $4^{\circ} \mathrm{C}$. The following day the brains were rinsed with PBS and stored in $30 \%$ sucrose in PBS for cryoprotection. Brains were sliced on a freezing microtome into $40 \mu \mathrm{m}$ coronal sections, and a one in six series of the dorsal hippocampus (approximately -1.5 to $-2.7 \mathrm{~mm}$ posterior to bregma; a total of six sections) was selected from each brain for immunofluorescent staining.

Immunofluorescent staining was performed as per the protocol from the laboratory of J.S. Rhodes (University of Illinois at Urbana-Champaign, Urbana-Champaign, IL; Mustroph et al., 2015). Free-floating sections were first washed with Tris-buffered saline (TBS). To denature DNA, the sections were then treated with a solution of $50 \%$ deionized formamide and $2 \times$ saline-sodium citrate for $2 \mathrm{~h}$ at $65^{\circ} \mathrm{C}$, washed in $2 \times$ saline-sodium citrate for $15 \mathrm{~min}$, treated with $2 \mathrm{~m}$ hydrochloric acid for $30 \mathrm{~min}$ at $37^{\circ} \mathrm{C}$, and washed with $0.1 \mathrm{~m}$ borate buffer for $10 \mathrm{~min}$. After a rinse in TBS, sections were blocked in a solution of TBS with $3 \%$ normal goat serum and $0.3 \%$ Triton X-100 (TBS-X) for 30 min before incubating in a primary antibody dilution in TBS- $X$ at $4^{\circ} \mathrm{C}$ for $48 \mathrm{~h}$. Sections were then washed with TBS, blocked in TBS-X for $30 \mathrm{~min}$, and incubated in a secondary antibody dilution in TBS-X for $3 \mathrm{~h}$. Primary antibodies used were rabbit anti-doublecortin (DCX; 1:250; Abcam; RRID:AB_732011) and mouse antineuronal-specific nuclear protein (NeuN; 1:500; Abcam; RRID:AB_10711040). Secondary antibodies used were Invitrogen donkey anti-rabbit and goat anti-mouse (all 1:250; Thermo Fisher Scientific; RRID:AB_141637 and RRID:AB_2535804) and were conjugated to Alexa Fluor 594 and 647 , respectively.

\section{Quantification and statistical analysis}

All sections were imaged on a confocal microscope with a $20 \times$ objective to visualize the entire granule cell layer of dentate gyrus in each of the six sections per animal. A z-stack of images was produced to encompass the complete thickness of the granule cell layer. Using NIS Elements software (Nikon), a maximum intensity projection was created in the $z$-plane to use for cell counting. The area of the granule cell layer and the number of $\mathrm{DCX}^{+}$ cells within the granule cell layer were calculated using a custom analysis in NIS Elements. The volume of each dentate gyrus section was calculated by multiplying this calculated area with the section thickness so that the average number of $\mathrm{DCX}^{+}$cells could be expressed per cubic micrometer of dentate gyrus.

All statistical analyses were performed with StatView, with $p<0.05$ considered to be statistically significant. Repeated-measures ANOVA was used to compare learning curves, with different groups as the independent variable and training day as the repeated measure. Unpaired $t$ tests were used to compare groups on each day of training, as well as the number of $\mathrm{DCX}^{+}$cells between the experimental and the combined control groups. Pearson's correlation coefficient was used to examine the relationship between the number of $\mathrm{DCX}^{+}$cells and various measures of learning. Outliers were removed if they exceeded 2 SDs beyond the mean; based on this criterion, one male animal was excluded from the experimental group. Data are expressed as the mean \pm SEM. A complete list of statistical tests and results can be found in Table 1.

\section{Results}

To ablate neural progenitor cells, Nes-TK mice were placed on a Val-infused diet 4 weeks before behavioral training. These mice were considered the experimental group, and both male and female mice exceeded the desired Val dosage of $200 \mathrm{mg} / \mathrm{kg} / \mathrm{d}(215 \pm 8.2$ and $235 \pm$ $9.3 \mathrm{mg} / \mathrm{kd} / \mathrm{d}$, respectively; Yu et al., 2008). The control 
Table 1. Statistics table

Figure 1: Comparison of learning curves

Male control groups

Female control groups

Male vs female controls

Male; Con vs Exp

Male; Con vs Exp

All sessions

Female; Con vs Exp

Figure 2: Comparing number of $\mathrm{DCX}^{+}$cells

Male; Con vs Exp

Female; Con vs Exp

Figure 3: Correlation between learning and number of $\mathrm{DCX}^{+}$cells Male; DCX vs average CRs

Male; DCX vs trials to 6 CRs of 10 trials

Female; DCX vs average CRs

Female; DCX vs trials to 6 CRs of 10 trials
Type of test

RM ANOVA

RM ANOVA

RM ANOVA

RM ANOVA

Unpaired $t$ tests

RM ANOVA

Unpaired $t$ test

Unpaired $t$ test

$\begin{array}{ll}\text { Pearson's correlation } & n=24 \\ \text { Pearson's correlation } & n=24 \\ \text { Pearson's correlation } & n=11 \\ \text { Pearson's correlation } & n=11\end{array}$

Pearson's correlation $n=24$

Con: $n=11$

Exp: $n=13$

Con: $n=6$

Exp: $n=5$

$\begin{array}{ll}\text { WT/Reg: } n=3 & \text { Group: } \\ \text { WT/Nal: } n=3 & F=3.84 \\ \text { Nes-TK/Reg: } n=5 & p=0.0677\end{array}$

WT/Reg: $n=3 \quad$ Group:

WT/Val: $n=1 \quad F=0.51$

Nes-TK/Reg: $n=2 \quad p=0.6454$

Male: $n=11$

Group:

$F=0.63$

$p=0.6346$

Exp: $n=13$

Con: $n=11$

Exp: $n=13$

Con: $n=6$

Exp: $n=5$

Group:

$F=15.2$

$p=0.0008$

Interaction:

$F=1.837$

$p=0.0487$

$\mathrm{H} 1: p=0.06$

$\mathrm{H} 2: p=0.24$

T1: $p=0.022$

T2: $p=0.0071$

T3: $p=0.0011$

$\mathrm{T} 4: p=0.029$

T5: $p=0.018$

T6: $p=0.0017$

T7: $p=0.0087$

T8: $p=0.0045$

T9: $p=0.034$

T10: $p=0.062$

Group:

$F=0.89$

$p=0.3696$

$p=0.0011$

$p=0.0947$

Pearson's correlation $\quad n=11$

$$
\begin{aligned}
& r=0.574 \\
& p=0.0027 \\
& r=-0.519 \\
& p=0.0121 \\
& r=-0.025 \\
& p=0.943 \\
& r=0.102 \\
& p=0.77
\end{aligned}
$$

All datasets are assumed to have normal distribution. Con, control; Exp, experimental; Reg, regular chow; Val, valganciclovir chow; RM, repeated measures.

groups consisted of Nes-TK mice eating regular chow, WT mice eating Val-chow, and WT mice eating regular chow.

All mice were trained on tEBC for $10 \mathrm{~d}$, with 40 trials per session. The EMG activity of the mice was monitored for CRs preceding US presentation, and an animal was considered to have reached learning criterion when it showed CRs for at least $60 \%$ of trials within any session. An example EMG trace of a well timed CR is shown in Figure $1 A$. There was no significant difference in learning among the three types of male controls $\left(F_{(2,8)}=3.84, p=0.0677\right)$, so they were combined into one control group, which is depicted in Figure $1 B$. The same was done for the female control group $\left(F_{(2,3)}=0.51, p=0.6454\right)$, shown in Figure 1C. Additionally, there was no significant difference in learning between the male and female control groups
$\left(F_{(1,15)}=0.63, p=0.6346\right)$. These two groups learned at approximately the same rate, reaching learning criterion on days 4 and 3 of training, respectively (Fig. 1B,C).

The male experimental group learned at a significantly slower rate than the male control animals $\left(F_{(1,22)}=15.2, p\right.$ $=0.0008)$, reaching learning criterion on day 10 of training (Fig. 1B). Additionally, there was a significant interaction between group and training day for male animals $\left(F_{(11,242)}\right.$ $=1.837, p=0.0487)$. Unpaired $t$ tests revealed that there was a significant difference between the experimental and control groups starting on the first day of training ( $p=$ $0.022)$, but this difference was no longer present by the final day of training ( $p=0.062)$. Training days 2 through 9 were also significantly different $(p<0.05)$, and a complete list of $p$ values can be found in Table 1. Female Nes-TK mice on the Val diet showed no difference in learning 
A

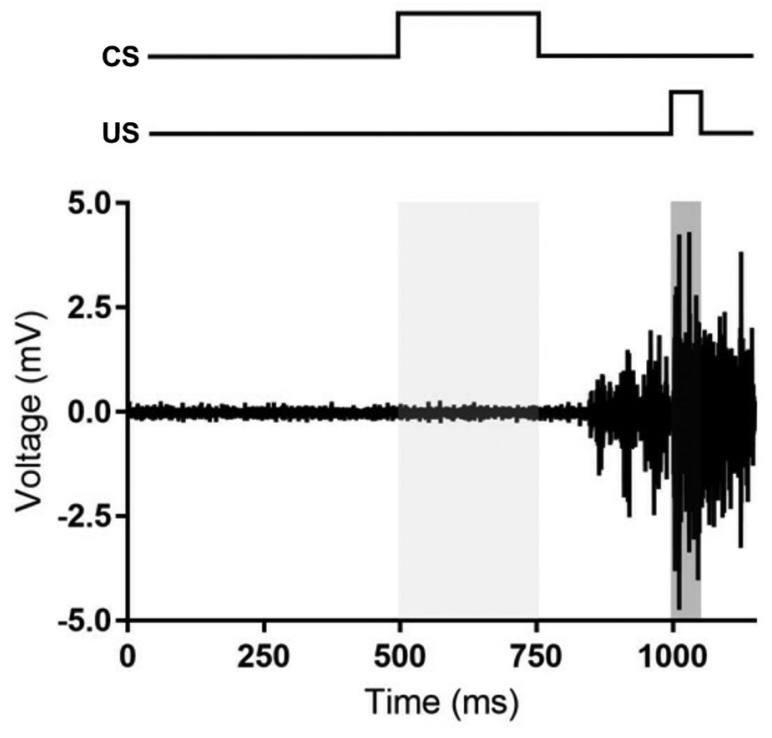

\section{B}

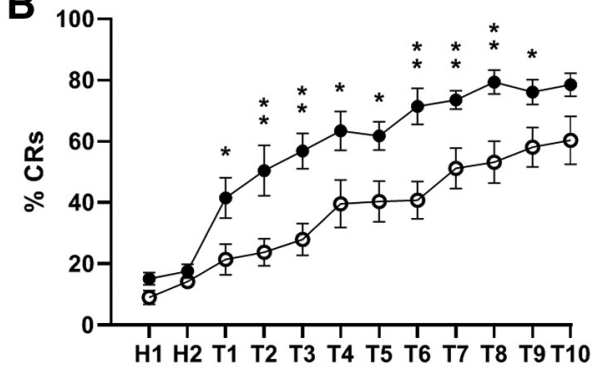

C

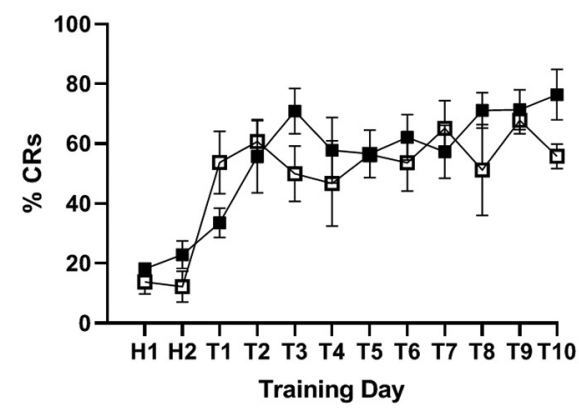

- Male Con $(n=11)$

- Male Exp (n=13)
- Female Con ( $n=6)$

๑ Female $\operatorname{Exp}(n=5)$

Figure 1. Trace eyeblink conditioning in mice. $\boldsymbol{A}$, EMG activity from an animal trained on tEBC (bottom trace), depicting a well timed $\mathrm{CR}$. The timing of the CS (whisker vibration) and US (air puff) presentation are shown at the top of the panel. $\boldsymbol{B}, \boldsymbol{C}$, Learning curves for the male $(\boldsymbol{B})$ and female $(\boldsymbol{C})$ control and experimental groups. Average percentage CRs are shown for each day, where " $\mathrm{H}$ " refers to days of habituation and "T" refers to days of training. Error bars represent the SEM. Post hoc $t$ tests were used to test statistical differences for each day of training $\left({ }^{*} p<0.05 ;{ }^{* *} p<0.01\right)$.

relative to the female control group $\left(F_{(1,9)}=0.89, p=\right.$ 0.3696; Fig. 1C).

Two weeks after the completion of training on tEBC, the number of $\mathrm{DCX}^{+}$cells in DG was quantified to validate the effect of valganciclovir on neurogenesis in Nes-TK mice (Fig. 2). DCX is a microtubule-associated protein ex- pressed by immature neurons that is used as a marker for neurogenesis (Brown et al., 2003; Couillard-Despres et al., 2005). The male experimental mice had significantly fewer $\mathrm{DCX}^{+}$cells relative to the male control group (7054 \pm 1529 and $16,754 \pm 2165$ cells $/ \mu \mathrm{m}^{3}$, respectively), showing a $58 \%$ decrease in the number of immature
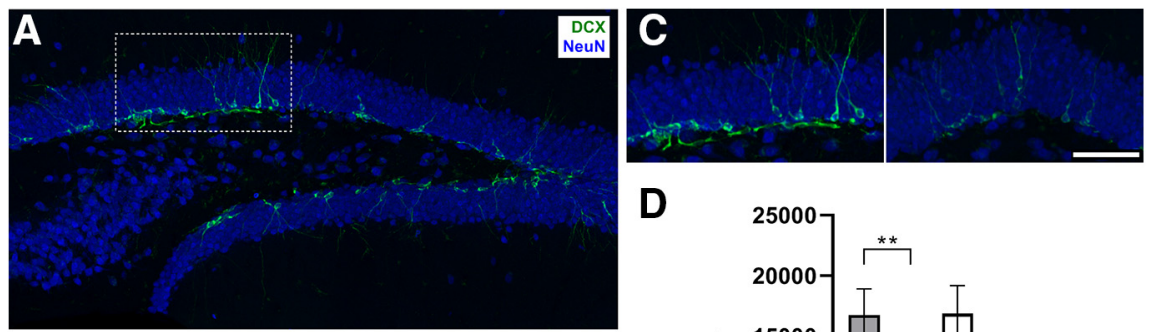

D
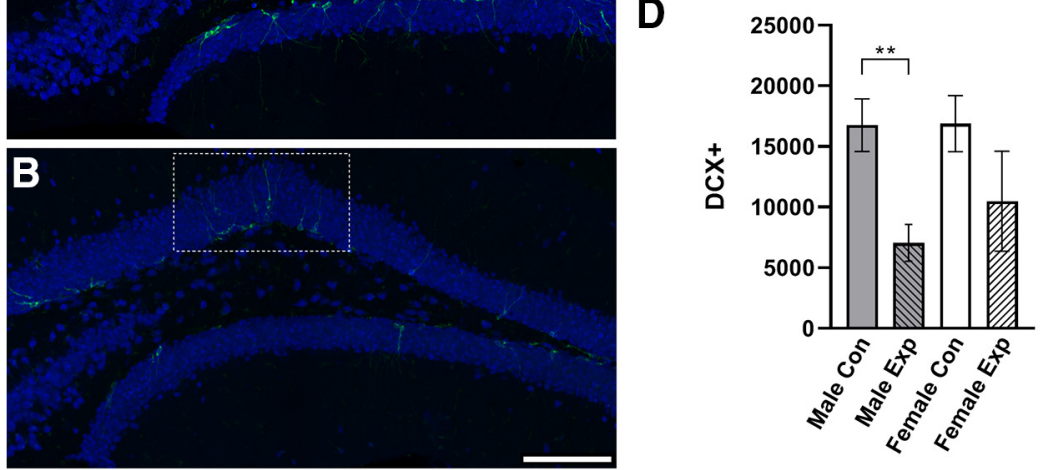

Figure 2. Measuring neurogenesis in the adult brain. $\boldsymbol{A}, \boldsymbol{B}$, Sample images of DCX expression in DG in sections from a male control animal $(\boldsymbol{A})$ and a male experimental animal $(\boldsymbol{B})$. $\boldsymbol{C}$, Zoomed in views of DCX ${ }^{+}$cells from $\boldsymbol{A}$ and $\boldsymbol{B}$ (left and right, respectively). $\boldsymbol{D}$, Quantification of the number of $\mathrm{DCX}^{+}$cells within the granule cell layer, expressed as the number of cells per cubic micrometer. The male experimental group $(n=13)$ showed a significant decrease relative to the male control group $\left(n=11 ;{ }^{* *} p<0.01\right)$. There was no significant difference between the female experimental group $(n=5)$ and the female control group $(n=6 ; p>0.05)$. Error bars represent the SEM. Scale bars: $\boldsymbol{B}, 100 \mu \mathrm{m} ; \boldsymbol{C}, 50 \mu \mathrm{m}$. 
A

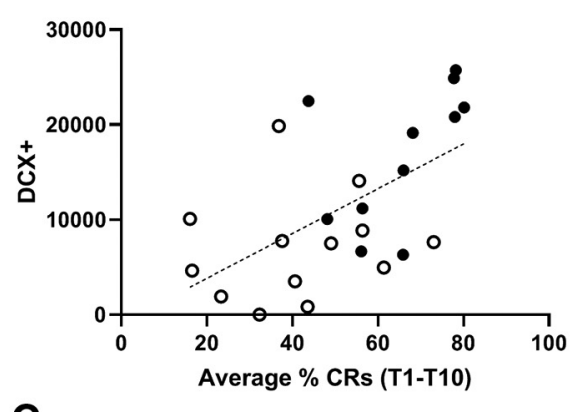

C

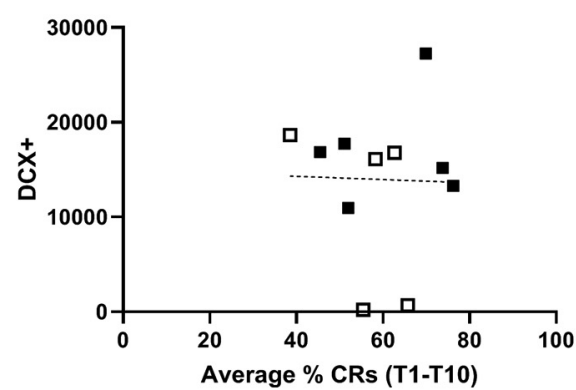

B

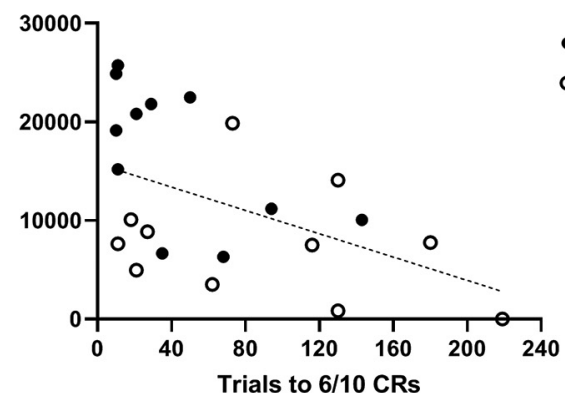

D

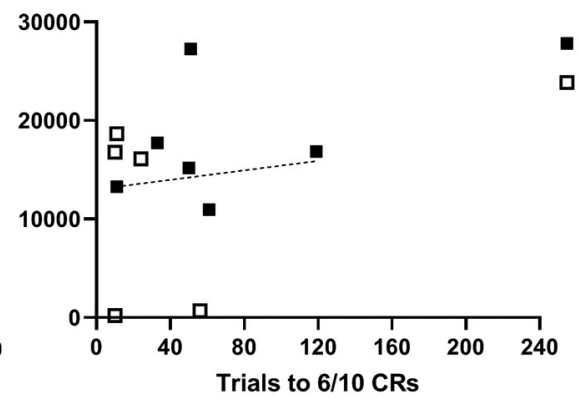

- Male Con

- Male Exp

ㅁemale Exp

Figure 3. The amount of neurogenesis is correlated with learning in male mice. $\boldsymbol{A}, \boldsymbol{C}$, The number of $\mathrm{DCX}^{+}$cells per micrometer of DG is positively correlated with the average percentage of CRs across all $10 \mathrm{~d}$ of training for males $(\boldsymbol{A} ; r=0.574, p=0.0027)$, but not females $(\boldsymbol{C} ; r=-0.025, p=0.943)$. $\boldsymbol{B}, \boldsymbol{D}$, The number of $\mathrm{DCX}^{+}$cells per cubic micrometer of $\mathrm{DG}$ is negatively correlated with the number of trials it took to show six CRs within a sliding block of 10 trials for males $(\boldsymbol{B} ; r=-0.519, p=0.0121)$, but not for females $(\boldsymbol{D} ; r=0.102, p=0.77)$.

neurons $(p=0.0011)$. Female experimental mice, however, only showed a nonsignificant $38 \%$ decrease relative to the female control group $(10,487 \pm 4120$ and $16,886 \pm$ 2304 cells $/ \mu \mathrm{m}^{3}$, respectively; $p=0.0947$; Fig. $\left.2 D\right)$.

A significant positive correlation was observed between the number of $\mathrm{DCX}^{+}$cells and the average percentage of CRs across all $10 \mathrm{~d}$ of training for male animals $(r=0.574$, $p=0.0027)$, indicating that male animals that had higher levels of neurogenesis performed better overall (Fig. $3 A$ ). Similarly, there was a significant negative correlation between the number of $\mathrm{DCX}^{+}$cells and the number of trials it took for a mouse to display six CRs within a sliding 10-trial block for male animals $(r=-0.519, p=0.0121)$, suggesting that male animals that had more newborn neurons learned at a faster rate (Fig. 3B). Female animals, however, showed no significant correlation between the number of $\mathrm{DCX}^{+}$cells and either of these measures of learning $(r=-0.025, p=0.943$; and $r=0.102, p=0.77$, respectively; Fig. $3 C, D$ ), although the Val diet may not have been as effective in our female mice as it was in our male mice.

\section{Discussion}

This study used genetic ablation of neural progenitor cells to explore whether adult neurogenesis is necessary for associative learning in mice. Ultimately, we found that decreasing neurogenesis led to an impairment in the acquisition of tEBC. Male Nes-TK mice on valganciclovir showed a nearly $60 \%$ reduction in the number of $\mathrm{DCX}^{+}$ cells and learned at a significantly slower rate than male control animals. Thus, newborn neurons are indeed in- volved in temporal associative learning, a finding that is in accordance with a previous study that used MAM to diminish the number of adult-born neurons in rats (Shors et al., 2001). Following this neurotoxic ablation of newborn neurons, Shors et al. (2001) observed a significant impairment in the ability of rats to learn tEBC. This previous study only eliminated newborn neurons born 1-2 weeks before training, while our study inhibited neurogenesis continuously before and during tEBC. However, our animals were able to eventually reach learning criterion by the end of training, unlike the results reported in the previous study where rats injected with MAM failed to reach the criterion. Our findings indicate that adult-born neurons contribute to learning this temporal association but are not the only dentate gyrus neurons that contribute. These other neurons are likely other types of dentate gyrus neurons or existing mature granule cells, neither of which were affected by our manipulation. The fact that the rats injected with MAM were unable to reach the learning criterion was likely due to nonspecific side effects of MAM, or possibly due to a species difference. It should be stressed that we observed impairment in learning starting on the very first training day while the previous study (Shors et al., 2001) did not, which further emphasizes the important role of adult-born neurons in learning.

In addition to an impairment in the rate of learning, we found that neurogenesis was correlated with various measures of learning in male mice. We observed a positive correlation between the number of $\mathrm{DCX}^{+}$cells and the average percentage of CRs across all $10 \mathrm{~d}$ of training, 
which suggests that male animals with more newborn neurons tended to perform better overall. This result is consistent with previous reports that demonstrated the same positive correlation between the number of newborn neurons and the average percentage CRs in rats trained on tEBC (Curlik and Shors, 2011). We also observed a significant negative correlation between the number of $\mathrm{DCX}^{+}$cells and the number of trials it took for male animals to display six CRs within a moving block of 10 trials, indicating that male animals that had higher levels of neurogenesis learned faster. This is the opposite of the correlation reported by Curlik and Shors (2011), who found that rats that took longer to learn tEBC showed a higher retention of neurons born before the beginning of training. These findings are not mutually exclusive, as Curlik and Shors (2011) injected bromodeoxyuridine before behavioral training to examine the survival of newborn neurons, while our study compared the overall production of adult-born neurons. Thus, while slower learning may be associated with increased survival of new neurons, our data suggest that animals with higher levels of neurogenesis learn at a faster rate. Interestingly, we observed no significant correlations between the number of $\mathrm{DCX}^{+}$cells and either measure of learning for female animals. This would suggest that females make use of alternative cell types or mechanisms to acquire tEBC and/or that females are less affected by the Val diet. Prior studies have demonstrated that not only do females use different strategies for spatial navigation, but that neurogenesis is differentially correlated with performance on a radial arm maze task depending on what strategy is used (Yagi et al., 2016).

Interestingly, we observed no behavioral effect in the Nes-TK female mice on valganciclovir. This is likely due to the fact that there was no significant reduction in neurogenesis relative to the female control group. It is possible that this manipulation was not effective in the females due to some degree of genetic drift during colony creation or maintenance, as other studies have used female Nes-TK mice with clear success (Hollands et al., 2017). This could also explain why we did not see as large a decrease in neurogenesis in the male animals as was observed in the initial study that used this transgenic line (Yu et al., 2008). Additionally, the male and female control groups learned at the same rate, unlike previous reports that found that female rats learned faster than males (Dalla et al., 2009). A possible reason for this disparity could be the difference in species, as the current study used mice while Dalla et al. (2009) used rats. Another possibility is the difference in the behavioral task, as the CS and US in the previous study were white noise and a shock to the eyelid, respectively, with a trace period of $500 \mathrm{~ms}$ (Dalla et al., 2009), while we used a $250 \mathrm{~ms}$ trace interval in the current study, with a whisker vibration CS and air puff US. With a longer trace period, and more trials needed to reach the learning criterion, the previous behavioral paradigm may have been more difficult to learn than the paradigm used in the current study. Therefore, it is also possible that we could observe a difference in the rate of learning between male and female mice if we used a longer trace interval. Regardless of these differences, our results clearly show in male mice that reduced neurogenesis in the dentate gyrus impairs the rate of hippocampal-dependent eyeblink conditioning.

Recent work by Suter et al. (2019) discovered cells within dentate gyrus that showed changes in the firing rate that started at CS onset and persisted through the trace period during tEBC. This finding suggests that there are cells within DG that are bridging the temporal gap between stimuli, which would play a vital role in associative learning. Because the cells that showed this persistent firing were highly excitable, the cell type was hypothesized to be either newborn neurons or mossy cells, as both are more excitable than granule cells (Mongiat et al., 2009; GoodSmith et al., 2017; Senzai and Buzsáki, 2017). Additionally, recent work by Madroñal et al. (2016) has demonstrated that while mature granule cells within DG are not involved in the retrieval of tEBC memory, transient inhibition of these cells led to a rapid, transient decrease in conditioned responses. This suggests that mature dentate granule cells are involved in the maintenance of associative memory. In the present study, we have demonstrated that adult-born neurons are indeed involved in acquiring tEBC, which provides a foundation to further explore exactly how newborn neurons and other cell types in dentate gyrus contribute to associative learning through in vivo recording techniques during behavioral training.

\section{References}

Altman J, Das GD (1965) Autoradiographic and histological evidence of postnatal hippocampal neurogenesis in rats. J Comp Neurol 124:319-335.

Brown JP, Couillard-Després S, Cooper-Kuhn CM, Winkler J, Aigner L, Kuhn HG (2003) Transient expression of doublecortin during adult neurogenesis. J Comp Neurol 467:1-10.

Couillard-Despres S, Winner B, Schaubeck S, Aigner R, Vroemen M, Weidner N, Bogdahn U, Winkler J, Kuhn HG, Aigner L (2005) Doublecortin expression levels in adult brain reflect neurogenesis. Eur J Neurosci 21:1-14.

Curlik DM, Shors TJ (2011) Learning increases the survival of newborn neurons provided that learning is difficult to achieve and successful. J C Neurosci 23:2159-2170.

Dalla C, Papachristos EB, Whetstone AS, Shors TJ (2009) Female rats learn trace memories better than male rats and consequently retain a greater proportion of new neurons in their hippocampi. Proc Natl Acad Sci U S A 106:2927-2932.

Dayer AG, Ford AA, Cleaver KM, Yassaee M, Cameron HA (2003) Short-term and long-term survival of new neurons in the rat dentate gyrus. J Comp Neurol 460:563-572.

Dupret D, Montaron MF, Drapeau E, Aurousseau C, Le Moal M, Piazza PV, Abrous DN (2005) Methylazoxymethanol acetate does not fully block cell genesis in the young and aged dentate gyrus. Eur J Neurosci 22:778-783.

Fritz AK, Amrein I, Wolfer DP (2017) Similar reliability and equivalent performance of female and male mice in the open field and watermaze place navigation task. Am J Med Genet 175:380-391.

GoodSmith D, Chen X, Wang C, Kim SH, Song H, Burgalossi A, Christian KM, Knierim JJ (2017) Spatial representations of granule cells and mossy cells of the dentate gyrus. Neuron 93:677-690.

Heiney SA, Wohl MP, Chettih SN, Ruffolo LI, Medina JF (2014) Cerebellar-dependent expression of motor learning during eyeblink conditioning in head-fixed mice. J Neurosci 34:14845-14853. 
Hollands C, Tobin MK, Hsu M, Musaraca K, Yu TS, Mishra R, Kernie SG, Lazarov O (2017) Depletion of adult neurogenesis exacerbates cognitive deficits in Alzheimer's disease by compromising hippocampal inhibition. Mol Neurodegener 12:64.

Lin C, Disterhoft J, Weiss C (2016) Whisker-signaled eyeblink classical conditioning in head-fixed mice. J Vis Exp. Advance online publication. Retrieved March 30, 2016. doi: 10.3791/53310.

Madroñal N, Delgado-García JM, Fernández-Guizán A, Chatterjee J, Köhn M, Mattucci C, Jain A, Tsetsenis T, Illarionova A, Grinevich V, Gross CT, Gruart A (2016) Rapid erasure of hippocampal memory following inhibition of dentate gyrus granule cells. Nat Commun 7:10923.

Madsen TM, Kristjansen PE, Bolwig TG, Wörtwein G (2003) Arrested neuronal proliferation and impaired hippocampal function following fractionated brain irradiation in the adult rat. Neuroscience 119:635-642.

M'Harzi M, Jarrard LE, Willig F, Palacios A, Delacour J (1991) Selective fimbria and thalamic lesions differentially impair forms of working memory in rats. Behav Neural Biol 56:221-239.

Mongiat LA, Espósito MS, Lombardi G, Schinder AF (2009) Reliable activation of immature neurons in the adult hippocampus. PLoS One 4:e5320.

Mustroph ML, Merritt JR, Holloway AL, Pinardo H, Miller DS, Kilby CN, Bucko P, Wyer A, Rhodes JS (2015) Increased adult hippocampal neurogenesis is not necessary for wheel running to abolish conditioned place preference for cocaine in mice. Eur $\mathrm{J}$ Neurosci 41:216-226.

Prendergast BJ, Onishi KG, Zucker I (2014) Female mice liberated for inclusion in neuroscience and biomedical research. Neurosci Biobehav Rev 40:1-5.

Saxe MD, Battaglia F, Wang JW, Malleret G, David DJ, Monckton JE, Garcia ADR, Sofroniew ER, Santarelli L, Hen R, Drew MR (2006) Ablation of hippocampal neurogenesis impairs contextual fear conditioning and synaptic plasticity in the dentate gyrus. Proc Natl Acad Sci U S A 103:17501-17506.
Senzai Y, Buzsáki G (2017) Physiological properties and behavioral correlates of hippocampal granule cells and mossy cells. Neuron 93:691-704.

Shors TJ, Miesegaes G, Beylin A, Zhao M, Rydel T, Gould E (2001) Neurogenesis in the adult is involved in the formation of trace memories. Nature 410:372-376.

Shors TJ, Townsend DA, Zhao M, Kozorovitskiy Y, Gould E (2002) Neurogenesis may relate to some but not all types of hippocampal-dependent learning. Hippocampus 12:578-584.

Snyder JS, Kee N, Wojtowicz JM (2001) Effects of adult neurogenesis on synaptic plasticity in the rat dentate gyrus. J Neurophysiol 85:2423-2431.

Snyder JS, Hong NS, McDonald RJ, Wojtowicz JM (2005) A role for adult neurogenesis in spatial long-term memory. Neuroscience 130:843-852.

Snyder JS, Choe JS, Clifford MA, Jeurling SI, Hurley P, Brown A, Kamhi JF, Cameron HA (2009) Adult-born hippocampal neurons are more numerous, faster-maturing and more involved in behavior in rats than in mice. J Neurosci 29:14484-14495.

Steckler T, Drinkenburg W, Sahgal A, Aggleton JP (1998) Recognition memory in rats-II. Neuroanatomical substrates. Prog Neurobiol 54:313-332.

Suter EE, Weiss C, Disterhoft JF (2019) Differential responsivity of neurons in perirhinal cortex, lateral entorhinal cortex, and dentate gyrus during time-bridging learning. Hippocampus 29:511-526.

Troncoso J, Múnera A, Delgado-García JM (2004) Classical conditioning of eyelid and mystacial vibrissae responses in conscious mice. Learn Mem 11:724-726.

Yagi S, Chow C, Lieblich SE, Galea L (2016) Sex and strategy use matters for pattern separation, adult neurogenesis, and immediate early gene expression in the hippocampus. Hippocampus 26:87101.

Yu TS, Zhang G, Liebl DJ, Kernie SG (2008) Traumatic brain injuryinduced hippocampal neurogenesis requires activation of early nestin-expressing progenitors. J Neurosci 28:12901-12912. 